\title{
Lectins from the Edible Mushroom Agaricus bisporus and Their Therapeutic Potentials
}

\author{
Wangsa Tirta Ismaya ${ }^{1}\left(\mathbb{D}\right.$, Raymond Rubianto Tjandrawinata ${ }^{1}\left(\mathbb{D}\right.$ and Heni Rachmawati ${ }^{2,3, *}$ \\ 1 Dexa Laboratories of Biomolecular Sciences, Dexa Medica, Industri Selatan V PP-7, Jababeka 2, \\ Cikarang 17550, Indonesia; wangsa.ismaya@dexa-medica.com (W.T.I.); raymond@dexa-medica.com (R.R.T.) \\ 2 School of Pharmacy, Bandung Institute of Technology, Ganesha 10, Bandung 40132, Indonesia \\ 3 Research Center for Nanosciences and Nanotechnology, Bandung Institute of Technology, Ganesha 10, \\ Bandung 40132, Indonesia \\ * Correspondence: h_rachmawati@fa.itb.ac.id; Tel.: +62-22-250-4852
}

Academic Editor: George Zervakis

Received: 22 March 2020; Accepted: 9 May 2020; Published: 20 May 2020

\begin{abstract}
The mushroom Agaricus bisporus secretes biologically active compounds and proteins with benefits for human health. Most reported proteins from A. bisporus are tyrosinases and lectins. Lectins are of therapeutic or pharmaceutical interest. To date, only limited information is available on A. bisporus lectins and lectin-like proteins. No therapeutic products derived from A. bisporus lectin (ABL) are available on the market despite its extensive exploration. Recently, A. bisporus mannose-binding protein $(\mathrm{Abmb})$ was discovered. Its discovery enriches the information and increases the interest in proteins with therapeutic potential from this mushroom. Furthermore, the A. bisporus genome reveals the possible occurrence of other lectins in this mushroom that may also have therapeutic potential. Most of these putative lectins belong to the same lectin groups as $\mathrm{ABL}$ and Abmb. Their relationship is discussed. Particular attention is addressed to ABL and Abmb, which have been explored for their potential in medicinal or pharmaceutical applications. ABL and Abmb have anti-proliferative activities toward cancer cells and a stimulatory effect on the immune system. Possible scenarios for their use in therapy and modification are also presented.
\end{abstract}

Keywords: anticancer; biological active fraction; immunomodulator; sugar-binding proteins; therapeutic protein

\section{Introduction}

Agaricus bisporus is one of the most consumed edible mushrooms in the world, and its benefit to human health has been widely reported. The mushroom is a popular part of the human diet. It is commonly called white mushroom, button mushroom, or champignon. A. bisporus is rich in metabolites and other biologically active compounds (amino acids, simple sugar/saccharides, indole, phenolic compounds, fatty acids, sterols, statins, vitamins, trace elements, and minerals), complex carbohydrates, and proteins [1]. In total, the mushroom fruiting bodies contain on average (non-dried weight) $\sim 30 \%$ proteins, $\sim 35 \%$ carbohydrates, $<5 \%$ others (sterol, saponin, tannin, terpenoid, minerals, and vitamins), and water (moisture) [2]. These constituents can be grouped into small and large molecules. The large molecules are proteins and complex carbohydrates. The mushroom is high in chitin content, thereby it is promoted for use as a source of dietary fiber. The small biologically active compounds and the proteins are associated with the anticancer, antiinflammation, antidiabetic, antihyperlipidemic, antioxidant, antiviral, and antimicrobial activities of the mushroom extract [1]. A. bisporus extracts inhibit the growth of epithelial and breast cancer cells as well as induce innate and adaptive immunity. Inclusion of the mushroom as part of the daily diet lowers the risk of breast cancer for postmenopausal women [3]. The anticancer activity is often referred to as an inhibition of 
aromatase activity by small compounds in the mushroom extracts or by immediate action of the lectin on the cancerous cells. These reports strengthen the attribute of the mushroom as a nutritional and remedial food.

The most studied proteins from $A$. bisporus are tyrosinase (also often called polyphenol oxidase, PPO) and lectin [4,5]. Indeed, bioactive proteins from edible mushrooms mostly comprise of lectin, ribosome-inactivating protein, copper oxygenase/oxidase (laccase, tyrosinase), antifungal proteins/peptides, and immunomodulatory proteins [6]. In this respect, it is important to note that ribosome-inactivating proteins are usually classified as lectin [7,8], which are known to display antifungal and immunomodulatory activities; hence, the antifungal and immunomodulatory proteins of the mushroom could also be grouped as lectin [9]. A. bisporus PPO and lectin are often employed in the search for human medicine, either as a bioactive molecule, as a molecular target, in the production of metabolites, or as a component in biochemical assays.

PPO catalyzes the conversion of L-tyrosine to L-3,4-dihydroxyphenylalanine (usually called L-DOPA), which is subsequently transformed into Dopaquinone, which is a precursor in melanin biosynthesis [4]. Melanin is a ubiquitous pigment in all organisms and PPO is associated with pigmentation in living organisms. PPO is mostly employed in the search for inhibitors against melanin formation, highly valuable in the search for skin whitening agents in the cosmetic industry and to prevent browning of agricultural produce [10]. The enzyme is also employed in the production of L-DOPA, the most effective agent in the treatment for Parkinson's disease [11], which is caused by impaired pigmentation in the brain by PPO and PPO-related proteins [12]. However, the direct use of PPO in therapy has been challenged because of its potential toxicity. PPO suppresses tumor growth, but it is also mutagenic [10]. The PPO catalyzed reaction generates unstable Dopaquinone that leads to the production of harmful oxy radicals, peroxides, semiquinones, and quinones [13], which are responsible for the antitumor activity of PPO, but also are neurotoxic [10]. The toxicity of the products and adducts generated by PPO result in a reluctance to use the enzyme directly in human therapy. Mushroom tyrosinase is commercially available and has long been employed primarily as a potent and viable inhibitor for anti-pigmentation, but not for therapeutic uses. Therefore, PPO and PPO-related proteins are not included in this study.

On the other hand, lectin is a protein with hemagglutinating (agglutination of red blood cells) activity. Its activity originates from the ability to recognize and reversibly bind carbohydrate and glycoconjugate (glycoprotein and glycolipid) [9]. Lectin is not an immunoglobulin, but is highly selective in and specific to the type of sugar/glycan, interacting with bound and free glycan, including monosaccharides (such as glucose, mannose, and galactose). Its interaction with the glycan is similar to that between antigen and antibody or enzyme and substrate [14]. The lectin's selectivity and specificity is the basis for its use in immunohematology, for example, as the marker for human blood typing [15]. The lectin family can be classified based on the structure of its carbohydrate recognition domain (CRD) or by the structural fold/feature. There are at least seven known groups of lectin: calnexin, L-type ( $\beta$-sandwich), P-type (rich in $\beta$-structures), C-type (mixed $\alpha / \beta$-structure), galectins ( $\beta$-sandwich), I-type (immunoglobulin superfamily fold), and R-type ( $\beta$-trefoil); the first three are intracellular, while the latter are extracellular [16]. The protein plays an important role in cellular processes, such as signaling, differentiation, and targeting [7]. Lectin is ubiquitous, but its occurrence and localization in tissues vary from one organism to another [17]. Most studies have been done with lectins from plants or animals, and only a limited number with lectins of fungal, bacterial, or viral origin. To date, only one lectin from $A$. bisporus has been reported, that is, A. bisporus lectin (ABL or ABA, A. bisporus agglutinin). Like mushroom tyrosinase, ABL is commercially available and has long been assessed for therapeutic use.

The use of $A$. bisporus in medication has mostly been studied using mushroom extracts, containing the small molecules (phenolic compounds, indoles, statins, and other secondary metabolites). For example, ergothioneine is an antioxidant associated with protection of monocytes activity, the indoles are associated with anticancer and anti-aging bioactivity, while ergosterol and ergocalciferol 
help to prevent vitamin D efficiency [1]. Commercial preparation of these compounds for therapeutic purposes includes complex extraction and isolation steps, while direct use of the extracts may be ineffective and undesirable. These small molecules are often present in a complex that is difficult to separate, whereas a long and complex extraction process may impact their stability and activity. Direct consumption of the fruiting bodies, that is, as part of the diet, might not be effective to achieve immediate therapeutic effect. On the other hand, the mushroom's bioactive fraction containing ABL has been reported to increase both insulin secretion by the pancreas and calcium uptake by the pancreatic islets up to three- and eleven-fold, respectively [18]. Thus, it is commercially attractive to produce mushroom lectins using molecular biology, that is, heterologous expression of the lectins using microbial cells as the host. Using that approach, modifications to the protein molecule can be introduced to improve its characteristics.

This paper focuses on lectins from A. bisporus, including ABL, with special attention to Abmb (A. bisporus mannose-binding protein), a recently discovered lectin-like protein with a unique characteristic. On its own, Abmb has no agglutination capacity, but it can display agglutinating activity of lectin when it occurs in complex with the mushroom PPO [19]. Thus, Abmb is unique in its relation to the other A. bisporus lectins with the similar $\beta$-trefoild (ricin B-like) fold. Nevertheless, its biological activity allows its inclusion as a therapeutic protein.

To date, only few proteins from $A$. bisporus have been investigated experimentally using an active fraction isolated from the fruiting bodies or as a recombinant protein. They are ABL [5], Abmb [20], PPO1 and PPO2 [21], PPO3 [4,22], PPO4 [23], and the most recently reported AB21 [24]. The latter is stable at a wide $\mathrm{pH}$ range (4.5-10.5), is thermostable, and has an affinity to transition metal ions, but displays no lectin activity. The AB21 structure consists of a helix bundle organized as collagen-like [24]. The AB21 structure resembles the animal mannose-binding protein, but AB21 occurs as a dimer instead of trimer. The biological relevance of AB21 is unclear at this moment.

\section{Lectins and Lectin-Like Protein in A. bisporus}

ABL is commercially available, and can even be provided in biotinylated, fluorescein isothiocyanante (FITC), and other fluorescence label-conjugated forms, mostly for bioanalysis and diagnosis (detection) purposes. The protein was discovered over 50 years ago and its therapeutic potential has been extensively studied. Yet, there is no therapeutic protein product derived from ABL for human applications on the market.

To date, only limited information is available on the lectins from A. bisporus. Since the A. bisporus genome was sequenced in 2012 [25], only one lectin from A. bisporus has been registered in the Genbank (ABL, GenBank ID XP_006455249). In 1995, two cDNA coding for ABL were cloned and the identity of the four ABL subunits in the tetrameric form was revealed [26]. At that time, gene products' isoformism was considered. Since the full genome of $A$. bisporus has been reported [25], no new lectins have been reported. The genomic data of $A$. bisporus $\mathrm{H} 97$ suggest the presence of 18 open reading frames (ORFs) that have been assigned as putative lectins. Two ORFs, Genbank ID of XP_006455555 and XP_006455253, were identified as Xerocomellus chrysenteron (red-cracking bolete mushroom) lectin (XCL) like proteins. The amino acid sequence of these two ORFs is highly homologous to ABL (Figure 1). However, as described later on (see 3.1), the isoformism of the subunits in ABL originates from variations in post-translational modification (glycoform), not from the merging of different gene products. 

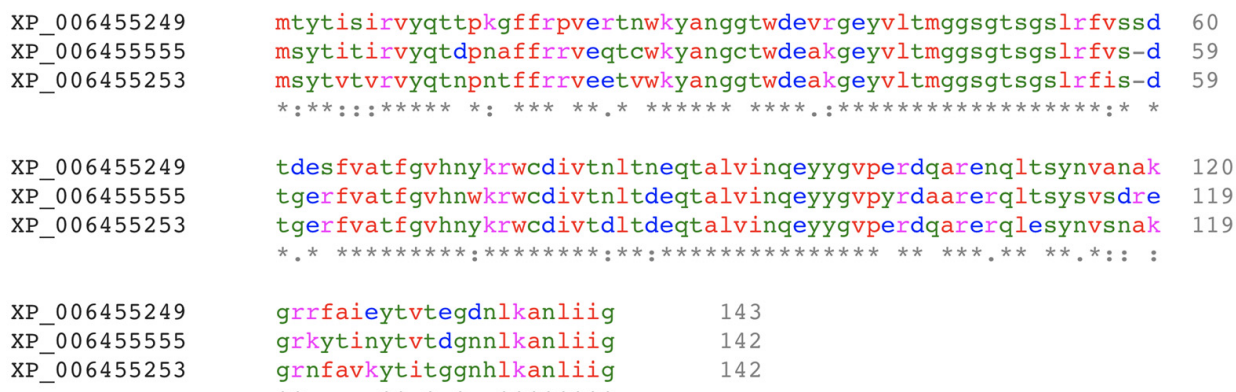

Figure 1. Alignment of amino acid sequences of A. bisporus lectin (ABL) (XP_006455249) and two of its closest putative lectin homologs in the A. bisporus U97 genomic library [27]. The protein annotations are according to the GenBank ID; the asterisk $(*)$, colon (:), and period (.) signs indicate the identical, conserved, and similar amino acid residues, respectively.

Next, two more ORFs, Genbank ID of XP_006460632 and XP_006461387, appear similar to lectin receptor kinase and legume-like lectin, respectively. Their similarity to the legume's lectin is not a surprise, considering the close relationship of fungal and plant lectins [28]. Four ORFs (Genbank ID of XP_006462966: 361 amino acids, XP_006459007: 414 amino acids, XP_006459008: 274 amino acids, and XP_006458911: 423 amino acids) encode proteins that contain the Ricin B-like lectin fold at either their N- or C-terminal. Proteins that contain a lectin domain are called chimerolectin [29] and occur commonly, for example, the Ricin B-like lectin domain of the fungal Marasmius oreades [30] and the C-domain of the fruit fly Drosophila melanogaster lectin [31].

Finally, the amino acid sequences of ten ORFs contain structural features of the Ricin B-like lectin fold (Genbank ID of XP_006456463: 162 amino acids, XP_006455093: 186 amino acids, XP_006463600: 172 amino acids, XP_006462967: 134 amino acids, XP_006463594: 163 amino acids, XP_006463593: 164 amino acids, XP_006462284: 148 amino acids, XP_006463575: 164 amino acids, XP_006463601: 160 amino acids, and XP_006455522: 171 amino acids). The amino acid sequences of five of the ten putative Ricin B-like lectins are homologous (Figure 2). Ricin B-like lectin has a typical $\beta$-trefoil fold [32], which is composed of a three-domain assembly with a pseudo-symmetry orientation and contains a $(\mathrm{Gln}-\mathrm{X}-\operatorname{Trp})_{3}$ sequence motif repeated in each of its domains [33,34].

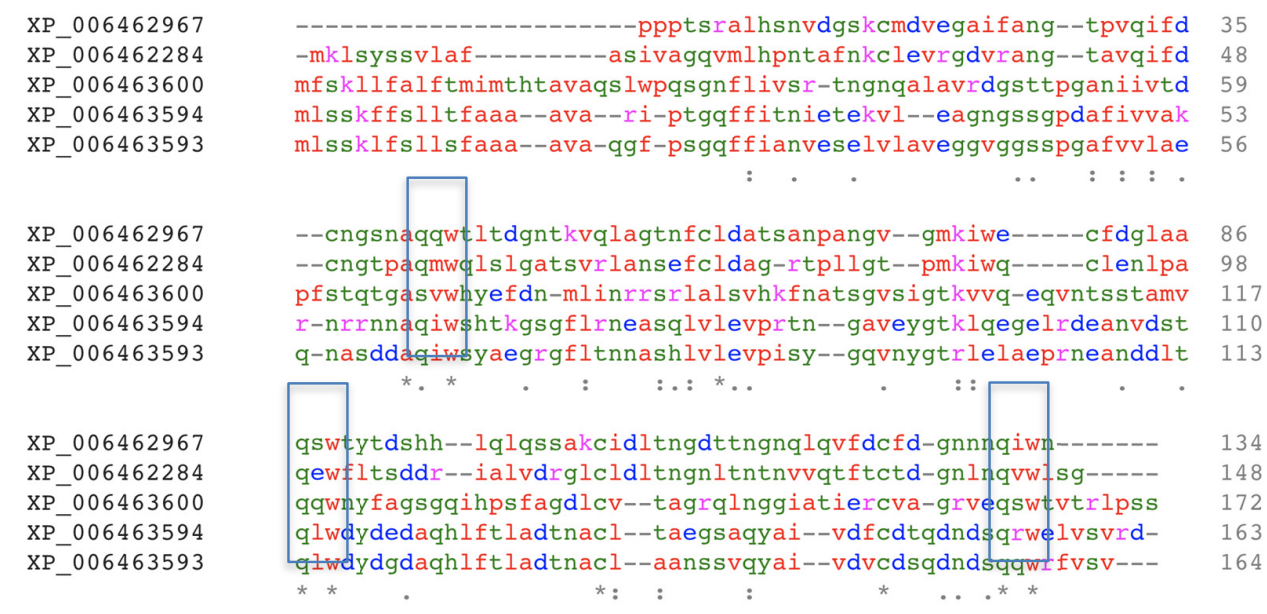

Figure 2. Alignment of amino acid sequences of the homologous putative Ricin B-like lectins [27]. The (Gln-x-Trp) 3 canonical sequence motifs of $\beta$-trefoil fold are highlighted in boxes. The protein annotations are according to the GenBank ID; the asterisk $\left(^{*}\right)$, colon $(:)$, and period (.) signs indicate the identical, conserved, and similar amino acid residues, respectively. 
On the basis of the carbohydrate binding module (CBM) database, lectins mostly contain CBM6, CBM13, CBM14, CBM18, CBM25, CBM26, CBM32, CBM36, CBM47, CBM57, and CBM60 [35]. In contrast, the $A$. bisporus genome indicates the potential presence of CBM1, CBM5, CBM13, CBM20, CBM21, and CBM35; where CBM1, CBM5, and CBM13 appear dominant [25]. CBM1 and CBM5 include lectins with affinity to complex carbohydrate, for example, cellulose [35] and possibly lignin [36], which is one of the major carbohydrate components in the mushroom. These CBM family members bind carbohydrates on the cellulose surface, so the binding site region in the protein surface is flat. Lectins from the CBM21 and CBM35 families bind to slightly less complex carbohydrate molecules, and have an affinity towards starches and xylan, respectively. Lectins from the CBM13 and CBM20 families bind oligosaccharides (four sugar units or more, non-branched) [35]. The CBM13 family includes lectin-like proteins and also binds short oligosaccharides (one to three sugar units).

Recently, a protein with a lectin-like structure was discovered [22] and named Abmb [37]. Interestingly, Abmb displays biological activities similar to lectins [20,38]. Abmb also has therapeutic potential [38]; hence, it is included in this review.

\section{Agaricus Bisporus Lectin (ABL)}

\subsection{Morphology, Characteristics, and Genetics}

In the early 1980s, ABL in mushroom extracts was found to be composed of two lectins, namely phytohemagglutinin-A and -B (PHA and PHB, respectively). Later, this mixture was found to contain four lectin isoforms (namely, I, II, III, and IV) with similar carbohydrate specificities and molecular weights [39]. ABL occurs as a $\sim 60 \mathrm{kDa}$ tetramer containing the four isoforms, presumably each weighing $\sim 16 \mathrm{kDa}$. These isoforms have different pI values $(5.53,5.69,5.98$, and 6.70$)$. Hence, they can be separated on a preparative isoelectric focusing chromatography column. The carbohydrate content analysis of the protein indicates that the four isoforms are glycoproteins [39]. The similarity of the four isoforms opens the possibility of variation in post-translational modification. Most importantly, the cDNA sequences of ABL isoforms obtained from a Southern blot analysis were identical. Further, the first 21 amino residues at the N-terminal of the four isoforms are also identical [26]. These results strongly suggest that the four ABL isoforms are derived from one gene product with variations in post-translational modification (glycoform). The isoforms with the most basic pI were successfully isolated and crystallized [40], which enabled the elucidation of the structure of ABL by X-ray crystallography.

ABL recognizes both galactose- $\beta-1,3-\mathrm{N}$-acetylgalactosamine (known as Thomsen-Friedenreich antigen or T-disaccharide) and galactose- $\beta-1,3-\mathrm{N}$-acetylglucosamine, but does not bind simple sugars (monosaccharides). ABL could also bind the sialylated form of the disaccharide (sialyl-2,3galactosil- $\beta-1,3-\mathrm{N}$-acetylgalactosamine) [41]. ABL agglutinates all erythrocytes independent of the blood group type. The type of sugar on the T-disaccharide recognized by ABL was characterized after treatment of erythrocytes with trypsin, which releases the O-linked glycopeptide, which completely abolishes ABL agglutination ability [39]. The ABL ability to agglutinate red blood cells originates from the presence of two distinct carbohydrate-binding sites on the ABL molecule [41], as confirmed by its crystal structure. ABL is classified as a hololectin, unlike R-type lectin (e.g., Abmb or its homologs), which is often found as a chimerolectin or merolectin [42].

From an evolutionary stand point, lectins with a similar structure to ABL are only found in fungi and archaeplastida from bryophytes (plants). The hypothesis is that the similarity of ABL with plant lectins is established via horizontal gene transfer upon endosymbiotics [28], thus fungal and plant lectins are evolutionarily related (Figure 3). However, the structural fold in ABL is unique and included as one of the twelve structural folds in plant lectins' classification; in a newer classification for plant lectins, ABL is grouped as a lectin fold that is specific to certain plant phyla [42].

In the A. bisporus genome, two other genes code for proteins similar to ABL (Figure 1). The presence of multiple genes coding for protein isoforms in A. bisporus appears a common feature, for example, there are six PPOs in A. bisporus, namely PPO1-PPO6. PPO1 is expressed constitutively, while PPO2 is 
inducible [21]. PPO1 and PPO6 are located at chromosome 8, while PPO2-PPO5 are at chromosome 5. Expression of these PPO isoforms appears to be correlated with different stages in the development of the mushroom fruiting bodies and their compartmentation [4]. So far, the nature of ABL gene expression and its gene products' compartmentation are not yet clear.

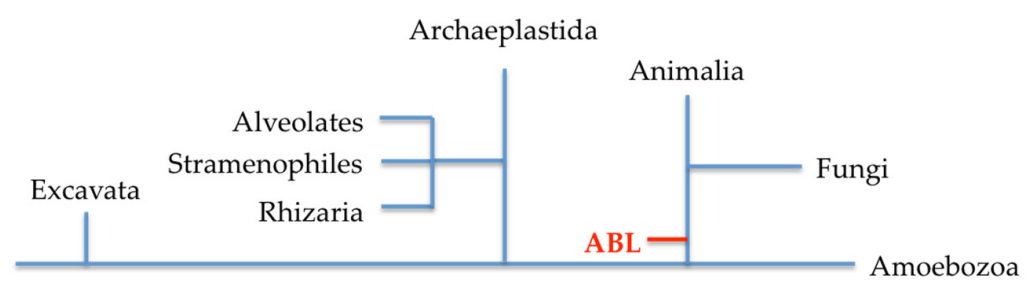

Figure 3. A phylogenetic relationship between fungal and plant lectins as presented by van Holle and van Damme [28].

\subsection{Structure and Possible Function in the Mushroom}

The crystal structure of ABL contains a $\beta$-sandwich fold, consisting of uneven pairing of two pleated four and six $\beta$-strands with two helices inserted in the long loop that connects the pleated sheet, forming an $\alpha$-loop- $\alpha$ motif (Figure $4 \mathrm{a}$ ). The tetrameric form is composed of a dimer of an ABL dimer, which is assembled similar to actinoporin (Figure 4b) [7], a pore-forming toxin that is commonly found in fungal fruiting-body lectins [43]. The two carbohydrate binding sites in each monomer are located at opposite positions in the protein molecule, but the monomers are assembled in the tetrameric form in such a way that all binding sites are accessible to the solvent [41]. This assembly allows independent binding of two sugar molecules in each monomer. Hence, ABL can display multivalent sugar-binding, and thereby agglutination. Each of the two sugar-binding sites in the ABL monomer is unique because each can distinguish two ligands differing only in the orientation of one hydroxyl group (epimer) [31].

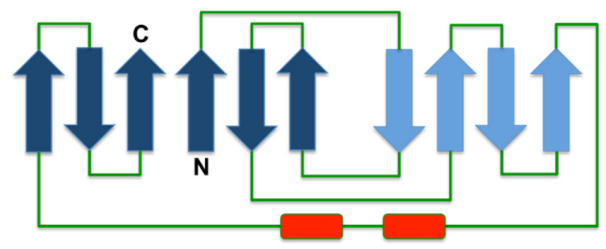

(a)

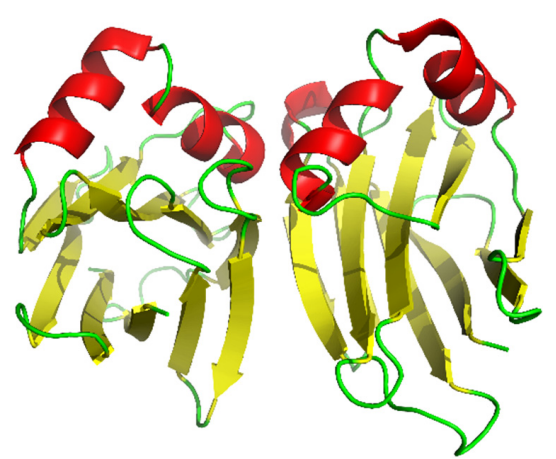

(b)

Figure 4. (a) Secondary structure topology of ABL. The pleated forms indicate the arrangement of the $\beta$-strands assembly. (b) Cartoon presentation of the crystal structure of ABL (Protein Data Bank (PDB) ID: 1Y2T). The structure was obtained from the Protein Databank and the three-dimensional structure figure was prepared using PyMoL [44]. The secondary structure topology was manually prepared using the three-dimensional structure as the reference. 
The binding of $\mathrm{N}$-acetylgalactosamine occurs at the first binding site, which involves Tyr28, His72, Tyr74, Tyr98, and Arg107, while N-acetylglucosamine is coordinated by Asp79, Thr82, Ile102, Arg103, Tyr114, and Val116 at the second binding site. The residues involved in the binding of sugars are strictly conserved in other lectins that have actinoporin fold, such as XCL, Neurosspora crassa (red bread mold) lectin, Pleurotus cornucopiae (golden oyster mushroom) lectin, Podospora anserina (mold of herbivore faeces) lectin (41], and Boletus edulis (penny bun mushroom) lectin [45]. The alignment of the amino acid sequences of XCL, N. crassa, P.cornucopiae, and P. anserina lectins reveals the Gly-Val-His-Asn-Tyr-Lys-X-Trp-X-Asp-Ile/Val-X-Thr/Val motif. About 14 amino acid before the first Gly in that motif, there is a Gly-X-X-X-Leu-X-X-X-Ser-Gly-Thr-Ser-Gly-Leu/Ile-Arg motif. Moreover, about 14 amino acid residues after the last Thr/Val, there is a X-Tyr-Tyr-X motif. Five other sugar-binding residues are located close to either the N- (X-Tyr-Ala-Asn-Gly) or C-terminal (X-Arg-X-X-X-Arg-X-X-Gln-X-X-X-Tyr) (amino acid residues participate in the binding of galactosyl and glucosyl sugars are in bold). In the 142 long amino acid sequence of ABL, most amino acid residues, composing the first binding site, reside in the first 70 residues (N-terminal half), while the second binding site is at the C-terminal half. Interestingly, these sugar-binding residues are completely conserved in XP_006455253 (Figure 1), while in XP_006455555, the Tyr in the -His-Asn-Tyrsequence motif is substituted with a Trp. However, it is unlikely that this substitution (Tyr74) alters the binding specificity or affinity, because this particular Tyr has minor contact with the sugar, providing a hydrophobic environment for the sugar ring to dock in the binding site [41]. Thus, so far, XP_006455555 and XP_006455253 could be predicted as the ABL isoforms with similar carbohydrate-binding activity.

Both ABL isoforms (GenBank ID of XP_006455555 and XP_006455253) are more basic (theoretical $\mathrm{pI}$ of 6.57 and 6.73, respectively; ABL is 5.85) and slightly more hydrophilic (GRAVY index of -0.542 and -0.531, respectively) than ABL (-0.529), likely because they contain less aliphatic side chains (Ala, Val, Ile, and Leu) (aliphatic index of 66.55 and 67.89, respectively; ABL is 68.11). This indicates that these putative $\mathrm{ABL}$ isoforms may be active in a different way in the mushroom or during development of the mushroom fruiting body. To date, very little is known about ABL function, on its compartmentation and localization of the encoding gene. Fungal lectins are postulated to function as a storage unit and/or a parasitic or infection agent. They also function in the development of fruiting bodies, morphogenesis, and defense mechanism. The latter is the most common proposed function, considering fungal lectin's nematodicidal and insecticidal properties [45].

\section{Agaricus bisporus Mannose-Binding Protein (Abmb)}

\subsection{Morphology, Characteristics, and Genetics}

$\mathrm{Abmb}$ was discovered as the light subunit of mushroom tyrosinase (PPO3) upon elucidation of the structure of the enzyme [22]. Abmb had been found consistently as an intrinsic part of the enzyme tetrameric complex [46], although its identity as a tyrosinase gene product had been questioned [47]. So far, separation of Abmb from the enzyme has not been successful, suggesting a strong and stable protein complex. Most importantly, this assembly of two proteins with different activity may indicate their synergistic functions in the mushroom.

$\mathrm{Abmb}$ is a non-glycosylated protein and migrates as an $\sim 18 \mathrm{kDa}$ species in a size exclusion chromatography column [38] (theoretical molecular weight of $16.5 \mathrm{kDa}$ ), thus in solution, it is monomeric. The protein has a $\mathrm{pI}$ value of $\sim 4.9$, as judged from an isoelectric focusing electrophoresis analysis [38] (theoretical pI is 5.34, thus, in actual condition, many of the negatively charged residues are buried). Abmb has a melting temperature at $50+1{ }^{\circ} \mathrm{C}$ and appears unstable at $\mathrm{pH}$ below 2.0. However, the protein resists proteolytic degradation by trypsin and papain, but not by bromelain [38]. During an absorption capability test using fresh jejunum, the full-length Abmb was digested, resulting in a $\sim 14 \mathrm{kDa}$ species in an SDS PAGE analysis [38]. Interestingly, this $\sim 14 \mathrm{kDa}$ species resembles the Abmb molecule that occurs as the tetrameric complex with PPO3. This tetrameric complex is 
consistently obtained from the commercial preparation, which is directly extracted from the mushroom fruiting bodies [22].

The Abmb encoding gene is located at chromosome 5, together with PPO (Figure 5a) [4]. Interestingly, despite its complex formation with PPO3, the location of the Abmb encoding gene sequence is closer to PPO5, one of the mushroom tyrosinase isoforms. The two genes are in opposite directions upon their translation. Abmb's highest gene expression occurs during the mycelium phase, early development of the flesh (stage 3), and development of the skin (stage 3-7). This Abmb gene expression correlates closely with that of PPO5. This is not a surprise considering their close co-localization on the chromosome [4].

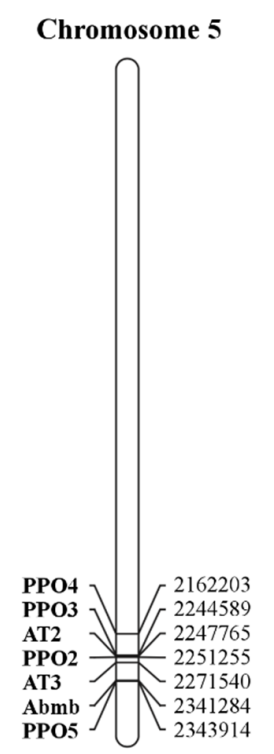

(a)

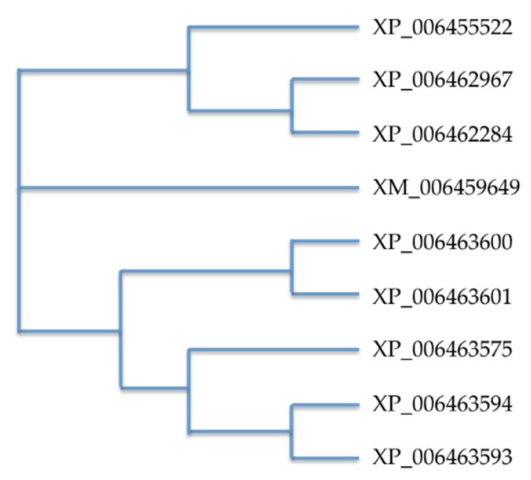

(b)

Figure 5. (a) The gene assembly in the region surrounding the Abmb encoding gene [4]. Numbers on the right refer to the position of the gene in the nucleotide genome sequence. AT stands for (4-hydroxy)phenylpyruvate amino transferase (b) Phylogenetic relationship between Abmb (GenBank ID XM_006459649) with other putative Ricin B-like lectins in A. bisporus. The phylogenetic tree was generated upon the amino acid sequence alignment using the program ClustalOmega [27]. PPO, polyphenol oxidase; Abmb, A. bisporus mannose-binding protein.

Abmb was not recognized as a lectin upon elucidation of the A. bisporus genome [25]. During an amino acid sequences alignment of ten ORFs coding for the putative Ricin-B like lectins, eight ORFs are similar Abmb. A phylogenetic tree analysis suggests that Abmb is not closely related to these eight 
ORFs. This indicates that Abmb may have a different function or activity from the eight ORFs. The two closest homologous ORFs to Abmb are XP_006463575 and XP_006455522, but their homology scores to $\mathrm{Abmb}$ are below $20 \%$. The sequence analysis even suggests that the Abmb structural homologs from the mushroom Clitocybe nebularis and the bacterium Clostridium botulinum are more closely related. This may be related to the sugar-binding characteristics of Abmb, because Ricin B-like lectins (lectins with $\beta$-trefoil fold) are galactose-binding proteins [48].

Most importantly, during a screening for sugar affinity by surface plasmon resonance analysis, $\mathrm{Abmb}$ was found to bind specifically to mannose, without any affinity for glucose, galactose, or fructose [37]. This result was confirmed by an in vitro analysis using MCF-7 and MDA-MB231 breast cancer cells. Abmb appears to be monomeric at higher concentrations and does not display the agglutination ability of lectin (tested up to 500 ppm $\sim 30 \mathrm{mM}$ ) [49], which is plausible as lectins with Ricin B-like structures with agglutination activity are oligomers in a complex with other proteins, or have a multivalent sugar-binding capacity. Abmb is predicted to have only one sugar-binding site that is similar to HA-33 and CNL, which are Abmb's closest structural homologs (see below) [50]. Recently, it has been demonstrated that Abmb participates in the agglutination of red blood cells when it occurs as a complex with the mushroom tyrosinase [19]. This strengthens the hypothesis that Abmb and PPO3 activities are synergistic and serve one function in the mushroom fruiting bodies. Most importantly, in the tetrameric complex with PPO3, Abmb is present in a mature form, in which one of the surface loops is proteolytically cleaved. The fact that $\mathrm{Abmb}$ is still able to cause agglutination suggests that the maturation has no impact on its sugar-binding capacity.

\subsection{Structure and Possible Function in the Mushroom}

The crystal structure of Abmb (PDB ID 5EHA) contains twelve $\beta$-strands organized in three domains, each of which contains two antiparalel $\beta$-hairpins. The Abmb domain architecture resembles that of Ricin B-like lectin, in which the first domain consists of $\beta 1, \beta 2$, and $\beta 3$ of the N-terminus and $\beta 12$ of the C-terminus, forming a classical Greek key motif. This is in line with the fact that Abmb's closest structural homologs are the hemagglutinin components of $C$. botulinum toxin (HA-33) and the Ricin B-like like lectin from C. nebularis (CNL) [22]. These two Abmb structural homologs share a similar carbohydrate-binding region and have a monovalent sugar binding mode; thus, the carbohydrate binding site of Abmb was postulated to resemble them [50]. However, HA-33 and CNL bind galactose; thereby, the sugar-binding fashion might differ, as demonstrated by a variation in the ABL sugar-binding sites. Indeed, the $\beta$-trefoil fold has been suggested to originate from a gene fusion event that links a 40-ish amino acid peptide with galactose-binding activity; each of these peptides becomes the subdomain [32]. Nevertheless, based on a phylogenetic study (Figure 5), Abmb is not closely related to any of the putative Ricin B-like lectins in A. bisporus.

The structure of monomeric full-length $\mathrm{Abmb}$ [51] shows very little deviation from the protein structure in the complex with PPO3 (Figure 6) [22]. A structural arrangement occurs at the N-terminal part, in which the flexible N-terminal rotates $90^{\circ}$ to expose an Abmb region that interacts with PPO3. The structure of the monomeric Abmb also shows the flexibility of a surface loop that is missing in the structure of Abmb in complex with PPO3 [51]. The sugar-binding in Abmb is similar to CNL, but the soaking of Abmb crystal with lactose, glucose, raffinose, or sucrose suggests that no interaction takes place with these sugars. The Abmb recognition of mannose (and mannitol), but not glucose (nor sorbitol), fructose, and galactose [37], provides a good explanation for the failed attempts to obtain $\mathrm{Abmb}$ crystals that contain lactose or glucose. Currently, an elucidation of the structure of Abmb in the presence of mannose and mannosyl sugars is on the way. 


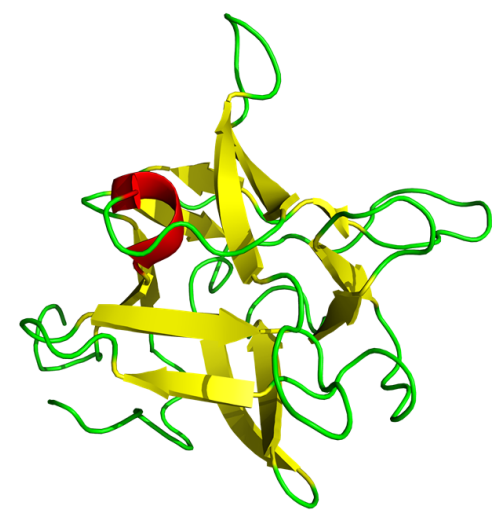

(a)

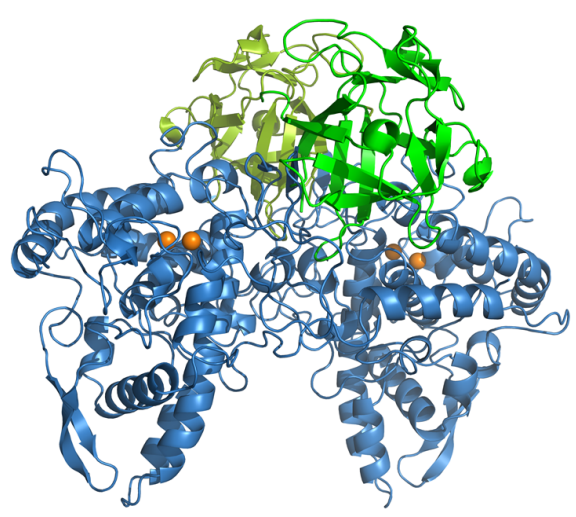

(b)

Figure 6. Cartoon presentation of the crystal structure of (a) Abmb (PDB ID: 5EHA) and (b) the tetrameric complex of PPO3-Abmb (PDB ID $2 \times 9$ Y). In the PPO3-Abmb complex, the tyrosinase is at the bottom, while the Abmb molecules are at the top. The structure was obtained from the Protein Databank. The three-dimensional structure figure was prepared using PyMoL [44].

The function of $\mathrm{Abmb}$ in the mushroom is not yet clear. However, the recent finding that $\mathrm{Abmb}$ in complex with PPO3 can execute agglutination may provide a hint on its biological function in the mushroom fruiting bodies [19]. A similar situation occurs for HA-33, which can only bind one sugar, but can display agglutination when in a hemagglutinin complex with HA-17, as part of the larger botulinum toxin complex containing HA-13 and HA-70 [52]. Thus, in mushrooms, assigning a lectin function to Abmb appears appropriate. PPO has an important role in the biosynthesis of melanins [4], which are bacteriostatic. Hence, the enzyme may play a role in a defense mechanism [53]. Abmb and PPO3 would be a synergic protein complex in the mushroom's defense mechanism. Abmb is most expressed during the mycelium stage and its presence remains high during the development stages of the fruiting bodies. Moreover, PPO5 is highly expressed in the mycelium stage and downregulated during the development of the fruiting bodies. PPO3 expression is regulated opposite to that of PPO5, thus PPO3 expression might compensate for the decreasing PPO5 expression [4]. It is possible that, during the transition from PPO5 to PPO3 and the down and up regulation of their expression, both PPO3 and Abmb undergo maturation and form the Abmb-PPO3 complex. Recruitment of PPO3 to form a complex with Abmb appears an obvious mechanism, because gene expression of PPO3 occurs in all fruiting body tissues (stem, flesh, and skin), except for in the gills (where PPO4 is present instead), at all development stages, ensuring that $\mathrm{Abmb}$ is present in most tissues. This hypothesis emphasizes the crucial role of Abmb in the mushroom defense mechanism. It is important to note that Abmb specificity towards mannose and mannitol (presumably also to mannosyl sugars) may complete the defense mechanism because the other lectins with $\beta$-trefoil in the mushroom $A$. bisporus are presumably galactose and/or glucose (and their derivatives) binding proteins.

\section{Potential Therapeutic Application of Lectin and Lectin-Like Protein from A. bisporus}

\section{1. $A B L$}

The potential use of ABL has been studied extensively for various therapeutic purposes. The first and best-known biological activity of ABL is its ability to inhibit proliferation of cancerous human epithelial colon cells (HT29) in vitro at a very low concentration $(50 \mu \mathrm{g} / \mathrm{mL} \sim 0.8 \mu \mathrm{M})$ [54]. The activity of ABL on colon cancer cells is likely derived from its ability to block the import of protein into the nucleus required for DNA synthesis during cell proliferation, directly blocking internal protein transport at the nuclear pores [5]. ABL also inhibits MCF-7 (breast cancer cells) and Caco-2 cancer cell proliferation in vitro, although at a lower potency [54]. Furthermore, the introduction of ABL to cultured human keratinocytes and papilloma transformed cells results in lower cell growth [55]. ABL slows down 
proliferation of human ocular fibroblast and reduces collagen lattice contraction in vitro, which is beneficial for wound healing of glaucoma patients after a trabeculectomy procedure [56]. At $90 \mu \mathrm{g} / \mathrm{mL}$ $(\sim 1.5 \mu \mathrm{M})$, ABL suppresses proliferation of retinal pigment epithelium (RPE) cells, which subsequently lowers the proliferative vitreoretinopathy and prevents redetachment of retinal cells after a surgical detachment procedure [57]. Moreover, exposure of rat's islet of Langerhans to ABL increases the conversion of pro-insulin to insulin, by enhanced proliferation of the pancreatic $\beta$-cell in vivo [58]. Thus, ABL might be employed in therapy for type- 1 and -2 diabetic patients who are suffering from a damaged pancreas. Finally, ABL also shows a strong inhibitory activity against human immunodeficiency virus type-1 (HIV-1) reverse transcriptase (IC50 of $8 \mu \mathrm{M}$ ) in vitro [59]. These reports show that ABL has a wide variety of cell targets and can be exploited for therapeutic applications as an anticancer, antidiabetic, and antiviral agent.

ABL has no cytotoxicity to normal cells [5]. However, ABL suppresses the immunoglobulin production in T and B lymphocyte cells through inhibition of DNA synthesis [60]. The introduction of ABL to the macrophage cells of mice, after stimulation with lipopolysaccharides, induces nitric oxide and the production of tumor necrosis factor (TNF)- $\alpha$, both in vitro and in vivo [61].

\subsection{Abmb}

Abmb can permeate a dialysis bag made of fresh jejunum ex vivo [20], and this ability is not altered upon its bioconjugation with a drug model [62]. This suggests that the protein might be employed as a drug carrier for oral administration. The use of $\mathrm{Abmb}$ as a drug carrier is supported by its resistance to the harsh gastrointestinal tract conditions [38]. Although Abmb internalization by the epithelial monolayer barrier of the intestine has not been described, generation of a species similar to mature $\mathrm{Abmb}$ (as in complex with PPO3) [38] indicates that Abmb undergoes proteolysis during the absorption, opening the possibility of transcellular permeability. In general, lectin absorption in the intestinal monolayer barrier is considered to occur via trans- or endocytosis [63]. At this moment, the mode of Abmb internalization by the intestinal cells is being studied.

At $12.5 \mu \mathrm{M}, \mathrm{Abmb}$ inhibits proliferation of MCF-7 breast cancer cells and arrests growth at lower concentrations in vitro [38]. The effect of Abmb administration at lower concentrations on MCF-7 cells proliferation does not impact the cell cycle, nor cell death. At higher concentrations, cell apoptosis occurs, as indicated by an increased expression of caspase-3, caspase-8, and caspase-9; upregulation of the pro-apoptosis protein $\mathrm{Bcl}-2$-associated X protein BAX; and downregulation of anti-apoptosis protein B-cell lymphoma-extra large Bcl-xL. Moreover, at low concentrations, Abmb activates expression of p53, which is important for genome stability by preventing genomic mutations [38]. At concentrations up to $6.25 \mu \mathrm{M}$, Abmb slightly increases proliferation of RAW 264.7 macrophage cells. Thus, the protein could potentially be recruited as an immunostimulator.

A preliminary immunogenicity test in Swiss Webster mice suggested that Abmb does not evoke a response of the immune system [20]. Further toxicity and immunogenicity tests with the inbreed and outbreed mice showed that Abmb was safe after prolonged administration at an elevated dose [64,65]. These results strengthen the safety profile of Abmb. This safety assessment is important for future use of Abmb.

\subsection{Potential Therapeutic Application from the Perspective of Other Lectins}

Lectins of plants, fungi, archaea, actinomycetes, and bacteria have been studied for their application as antiviral agents [66]. Lectin's potencies as an antiviral agent have been shown against a variety of viruses, for example, herpes simplex type 1 and 2, hepatitis C, influenza A/B, HIV, Japanese encephalitis virus, and the current Coronavirus Covid-19; the effective concentration range is $1.6 \mathrm{nM}-1.3 \mu \mathrm{M}$ (see [66] and the references therein). The potential use as an anticancer agent has also been widely studied with various cancerous cells such as epidermal keratinocyte, lung, thyroid, [67], fibroblast, colon, prostate, colorectal, melanocyte, liver, mesenchyma, lymphoid, brain, and spinal (and the 
references therein). Most of these potentials therapeutic applications have been tested with ABL (see above), but not with Abmb, because of its ambiguous function and activity.

\section{Future Development of Abmb}

ABL is a potent anticancer agent, but its development as such has halted in the last decade. Instead, other possible applications in diabetes therapy or modulating the immune system emerged. The concentration of $\mathrm{Abmb}$ required to produce a significant effect on cancer cells is much higher than with ABL. Thus, Abmb development as an anticancer agent may require further assessment and possibly modification of the protein molecule.

At lower concentrations, Abmb might be useful for other purposes, that is, targeted drug carrier or complementing the immune system, taking the advantage of Abmb's (and its bioconjugate) ability to permeate the jejunum and its specific affinity to mannose, respectively. Abmb could be conjugated to an anti-breast cancer drug, making sure the drug is directed to the cancer cell target (Figure 7). Upon attachment to the receptor on the cell surface, the anticancer drug load could be released and Abmb goes on to circulate in the blood stream, or the bioconjugate as a whole undergoes endocytosis. The latter is a plausible proposition based on Abmb's ability to initiate p53 expression. In this instance, harnessing p53 role in cell apoptosis and senescence has been considered as the most useful cancer therapy [68]. Therefore, elucidation of Abmb's exact mechanism in activating the p53 expression is crucial. The strategy to bring an anticancer drug into the cell target has been extensively developed. As an example, mannose, decorated on the surface of nano particles containing doxorubicin, has been successfully demonstrated to deliver the drug into breast cancer cells [69]. This strategy exploits high expression of mannose receptors on the surface of human malignant tumor cells. Though the nature of $\mathrm{Abmb}$ interaction with the breast cancer cells is not yet known, the Abmb specificity towards this type of cancer cell could be exploited.

Interestingly, breast cancer chemotherapy using the combination of doxorubicin and cyclophosphamide is reported to have a negative impact because the mannose-binding lectin 2 (MBL2) expression in breast cancer patients receiving this combination of drugs is downregulated [70]. Lower expression of MBL2 leads to higher risk of grade 3 infection from a compromised immune system condition. Introduction of Abmb into the blood circulation might compensate downregulation of MBL2, slow down breast cancer cell growth, and stimulate the immune system. Abmb could be administered via oral administration, benefiting from its absorbability in the intestine. Recently, a strategy to deploy magnetic beads coated with the fusion of the MBL and Fc domain of the antibody has been successfully developed to capture circulating tumor cells, mimicking the opsonization mechanism of the innate immune system [71]. This strategy could be adopted to further Abmb functionalization.

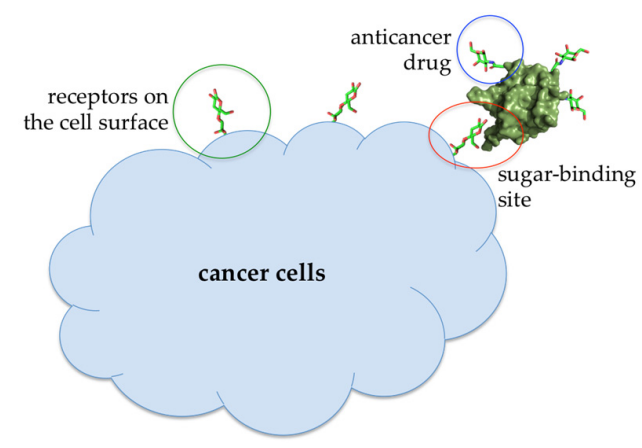

Figure 7. Illustration of an Abmb bioconjugate carrying an anticancer drug upon recognition by a receptor (presumably a sugar) on the cell surface. 


\section{Conclusions}

Lectins and lectin-like protein from the mushroom A. bisporus hold a great potential for application in health and medicine. Lectins can be consumed as part of the diet or economically produced either as protein extracts (from cheaply and easily cultivated mushroom) or as recombinant proteins for specific targeting in medicinal or pharmaceutical application.

Author Contributions: Conceptualization, W.T.I.; Resources, R.R.T.; Writing-Original Draft Preparation, W.T.I.; Writing_-Review \& Editing, H.R.; Supervision, R.R.T.; Funding Acquisition, H.R. All authors have read and agreed to the published version of the manuscript.

Funding: The works leading to this review have been funded by the Ministry of Research Technology and Higher Education - Republic of Indonesia under the World Class Research grant managed by Institut Teknologi Bandung, and by Dexa Medica.

Acknowledgments: We thank J.J. Beintema from the Biochemistry laboratory and B.W. Dijkstra from the Protein X-ray Crystallography group at the University of Groningen, The Netherlands, for the insights and inspiration in the biochemistry and structural study of Abmb and A. bisporus PPO. We also thank H.J. doddema for the editing of the manuscript.

Conflicts of Interest: The authors declare no conflict of interest.

\section{References}

1. Muszyńska, B.; Kała, K.; Rojowski, J.; Grzywacz, A.; Opoka, W. Composition and biological properties of Agaricus bisporus fruiting bodies-A Review. Pol. J. Food Nutr. Sci. 2017, 67, 173-181. [CrossRef]

2. Eswari, S.; Saravana Bhavan, P.; Kalpana, R.; Dharani, C.; Manjula, T.; Sarumathi, K.; Rajkumar, G. Phytochemical characterization of the mushroom Agaricus bisporus and assessment of its nutritional ability in the place of fishmeal for survival and growth of the freshwater prawn Macrobrachium rosenbergii post-larvae. Integr. Food Nutr. Metab. 2019, 6, 1-11.

3. Dhamodharan, G.; Mirunalini, S. A Novel Medicinal Characterization of Agaricus Bisporus (White Button Mushroom). Pharmacologyonline 2010, 2, 456-463.

4. Weijn, A.; Bastiaan-Net, S.; Wichers, H.J.; Mes, J.J. Melanin biosynthesis pathway in Agaricus bisporus mushrooms. Fungal Genet. Biol. 2013, 55, 42-53. [CrossRef] [PubMed]

5. Yu, L.G.; Fernig, D.G.; White, M.R.; Spiller, D.G.; Appleton, P.; Evans, R.C.; Grierson, I.; Smith, J.A.; Davies, H.; Gerasimenko, O.V.; et al. Edible mushroom (Agaricus bisporus) lectin, which reversibly inhibits epithelial cell proliferation, blocks nuclear localization sequence-dependent nuclear protein import. J. Biol. Chem. 1999, 274, 4890-4899. [CrossRef] [PubMed]

6. Xu, X.; Yan, H.; Chen, J.; Zhang, X. Bioactive proteins from mushrooms. Biotechnol. Adv. 2011, 29, 667-674. [CrossRef]

7. Hassan, M.A.A.; Rouf, R.; Tiralongo, E.; May, T.W.; Tiralongo, J. Mushroom lectins: Specificity, structure and bioactivity relevant to human disease. Int. J. Mol. Sci. 2015, 16, 7802-7838. [CrossRef]

8. Walsh, M.J.; Dodd, J.E. Hautbergue GM. Ribosome-inactivating proteins: Potent poisons and molecular tools. Virulence 2013, 4, 774-784. [CrossRef]

9. Santos, A.F.S.; Silva MDCd Napoleaão, T.H.; Paiva, P.M.G.; Correia, M.T.S.; Coelho, L.C.B.B. Lectins: Function, structure, biological properties and potential applications. Curr. Top. Pep. Protein Res. 2014, 15, 41-62.

10. Seo, S.-Y.; Sharma, V.K.; Sharma, N. Mushroom Tyrosinase: Recent Prospects. J. Agric. Food Chem. 2003, 51, 2837-2853. [CrossRef]

11. Muthuraman, M.; Koirala, N.; Ciolac, D.; Pintea, B.; Glaser, M.; Groppa, S.; Tamás, G.; Groppa, S. Deep brain stimulation and L-DOPA therapy: Concepts of action and clinical applications in Parkinson's disease. Front. Neurol. 2018, 9, 711. [CrossRef] [PubMed]

12. Tief, K.; Schmidt, A.; Beermann, F. New evidence for presence of tyrosinase in substantia nigra, forebrain and midbrain. Brain Res. Mol. Brain Res. 1998, 53, 307-310. [CrossRef]

13. Graham, D.G. Oxidative pathways for catecholamines in the genesis of neuromelanin and cytotoxic quinones. Mol. Pharmacol. 1978, 14, 633-643. [PubMed]

14. Ghazarian, H.; Idoni, B.; Oppenheimer, S.B. A glycobiology review: Carbohydrates, lectins, and implications in cancer therapeutics. Acta Histochem. 2011, 113, 236-247. [CrossRef] [PubMed] 
15. Gorakshakar, A.C.; Ghosh, K. Use of lectins in immunohematology. Asian J. Tranfus. Sci. 2016, 10, $12-21$. [CrossRef] [PubMed]

16. Dodd, R.B.; Drickamer, K. Lectin-like proteins in model organisms: Implications for evolution of carbohydrate-binding activity. Glycobiology 2001, 11, 71R-79R. [CrossRef]

17. Khan, F.; Khan, M.I. Fungal lectins: Current molecular and biochemical perspectives. Int. J. Biol. Chem. 2011, 5, 1-20. [CrossRef]

18. Ahmad, N.; Bansal, R.; Rastogi, A.K.; Kidwai, J.R. Effect of PHA-B fraction of Agaricus bisporus lectin on insulin release and 45Ca2+ uptake by islets of Langerhans in vitro. Acta Diabetol. Lat. 1984, 21, 63-70. [CrossRef]

19. Ismaya, W.T.; Tjandrawinata, R.R.; Dijkstra, B.W.; Beintema, J.J.; Rachmawati, R.R. Relationship of Agaricus bisporus mannose-binding protein to lectins with $\beta$-trefoil fold. Biochem. Biophys. Res. Commun. 2020. In Press. [CrossRef]

20. Ismaya, W.T.; Yunita, E.A.; Lai, X.; Retnoningrum, D.S.; Rachmawati, H.; Dijkstra, B.W.; Tjandrawinata, R.R. A novel immune-tolerable and permeable lectin-like protein from mushroom Agaricus. Bisporus. Biochem. Biophys Res. Commun. 2016, 473, 1090-1093. [CrossRef]

21. Wichers, H.J.; Recourt, K.; Hendriks, M.; Ebbelaar, C.E.M.; Biancone, G.; Hoeberichts, F.A.; Mooibroek, H.; Soler-Rivas, C. Cloning, expression and characterisation of two tyrosinase cDNAs from Agaricus bisporus. Appl. Microbiol. Biotech. 2003, 61, 336-341. [CrossRef] [PubMed]

22. Ismaya, W.T.; Rozeboom, H.J.; Weijn, A.; Mes, J.J.; Fusetti, F.; Wichers, H.J.; Dijkstra, B.W. Crystal structure of Agaricus bisporus mushroom tyrosinase: Identity of the tetramer subunits and interaction with tropolone. Biochemistry 2011, 50, 5477-5486. [CrossRef] [PubMed]

23. Mauracher, S.; Molitor, C.; Michael, C.; Kragl, M.; Rizzi, A.; Rompel, A. High level protein-purification allows the unambiguous polypeptide determination of latent isoform PPO4 of mushroom tyrosinase. Phytochemistry 2014, 99, 14-25. [CrossRef] [PubMed]

24. Komárek, J.; Kavková, E.I.; Houser, J.; Horáčková, A.; Ždánská, J.; Demo, G.; Wimmerová, M. Structure and properties of AB21, a novel Agaricus bisporus protein with structural relation to bacterial pore-forming toxins. Proteins Struct. Func. Bioinf. 2018, 86, 897-911. [CrossRef] [PubMed]

25. Morin, E.; Kohler, A.; Baker, A.R.; Foulongne-Oriol, M.; Lombard, V.; Nagye, L.G.; Ohm, R.A. Genome sequence of the button mushroom Agaricus Bisporus reveals mechanisms governing adaptation to a humic-rich ecological niche. Proc. Natl. Acad. Sci. USA 2012, 109, 17501-17506. [CrossRef] [PubMed]

26. Crenshaw, R.W.; Harper, S.N.; Moyer, M.; Privalle, L.S. Isolation and Characterization of a cDNA Clone Encoding a Lectin Gene from Agaricus bisporus. Plant Physiol. 1995, 107, 1465-1466. [CrossRef]

27. Sievers, F.; Wilm, A.; Dineen, D.; Gibson, T.J.; Karplus, K.; Li, W.; Lopez, R.; McWilliam, H.; Remmert, M. Fast, scalable generation of high-quality protein multiple sequence alignments using Clustal Omega. Mol. Syst. Biol. 2011, 7, 539. [CrossRef]

28. Van Holle, S.; Van Damme, E.J.M. Messages from the past: New insights in plant lectin evolution. Front. Plant Sci. 2019, 10, 1-14. [CrossRef]

29. Barre, A.; Bourne, Y.; Van Damme, E.J.M.; Rougé, P. Overview of the structure-function relationships of mannose-specific lectins from plants, algae and fungi. Int. J. Mol. Sci. 2019, 20, 254. [CrossRef]

30. Grahn, E.; Askarieh, G.; Holmner, A.; Tateno, H.; Winter, H.C.; Goldstein, I.J.; Krengel, U. Crystal structure of the marasmius oreades mushroom lectin in complex with a xenotransplantation epitope. J. Mol. Biol. 2007, 369, 710-721. [CrossRef]

31. Ji, S.; Samara, N.L.; Revoredo, L.; Zhang, L.; Tran, D.T.; Muirhead, K.; abak, L.A.; Ten Hagen, K.G. A molecular switch orchestrates enzyme specificity and secretory granule morphology. Nat. Commun. 2018, 9, 1-13. [CrossRef] [PubMed]

32. Cummings, R.D.; Schnaar, R.L. R-Type Lectins. In Essentials of Glycobiology, 3rd ed.; A Varki, R.D.C., Esko, J.D., Stanley, P., Hart, G.W., Aebi, M., Darvill, A.G., Kinoshita, T., Packer, N.H., Prestegard, J.H., Schnaar, R.L., Seeberger, P.H., Eds.; Cold Spring Harbor: New York, NY, USA, 2017.

33. Murzin, A.G.; Lesk, A.M.; Chothia, C. Beta-Trefoil fold. Patterns of structure and sequence in the Kunitz inhibitors interleukins- 1 beta and 1 alpha and fibroblast growth factors. J. Mol. Biol. 1992, 223, 531-543. [CrossRef]

34. Hazes, B. The (QxW)3 domain: A flexible lectin scaffold. Prot. Sci. 1996, 5, 1490-1501. [CrossRef] [PubMed] 
35. CAZypedia, T.C. Ten years of CAZypedia: A living encyclopedia of carbohydrate-active enzymes. Glycobiology 2017, 28, 3-8.

36. Yarbrough, J.M.; Mitta, A.; Mansfield, E.; Taylor, L.E.; Hobdey, S.E.; Sammond, D.W.; Bomble, Y.J.; Crowley, M.F.; Decker, S.R.; Himmel, M.E.; et al. New perspective on glycoside hydrolase binding to lignin from pretreated corn stover. Biotechnol. Biofuels. 2015, 8, 214. [CrossRef]

37. Rachmawati, H.; Sundari, S.; Nabila, N.; Tandrasasmita, O.M.; Amalia, R.; Siahaan, T.J.; Tjandrawinata, R.R.; Ismaya, W.T. Orf239342 from the mushroom Agaricus bisporus is a mannose binding protein. Biochem. Biophys. Res. Commun. 2019, 515, 99-103. [CrossRef]

38. Ismaya, W.T.; Tandrasasmita, O.M.; Sundari, S.; Lai, X.; Retnoningrum, D.S.; Dijkstra, B.W.; Tjandrawinata, R.R.; Rachmawati, H. The light subunit of mushroom Agaricus bisporus tyrosinase: Its biological characteristics and implications. Int. J. Biol. Macromol. 2017, 102, 308-314. [CrossRef]

39. Sueyoshi, S.; Tsuji, T.; Osawa, T. Purification and characterization of four isolaectins of mushroom (Agaricus bisporus). Biol. Chem. 1985, 366, 213-221.

40. Carrizo, M.E.; Irazoqui, F.J.; Lardone, R.D.; Nores, G.A.; Curtino, J.A.; Capaldi, S.; Perduca, M.; Monaco, H.L. Crystallization and preliminary X-ray study of the common edible mushroom (Agaricus bisporus) lectin. Acta Crystallogr. 2004, D60, 718-720. [CrossRef]

41. Carrizo, M.E.; Capaldi, S.; Perduca, M.; Irazoqui, F.J.; Nores, G.A.; Monaco, H.L. The antineoplastic lectin of the common edible mushroom (Agaricus bisporus) has two binding sites, each specific for a different configuration at a single epimeric hydroxyl. J. Biol. Chem. 2005, 280, 10614-10623. [CrossRef]

42. Jiang, S.; Ma, Z.; Ramachandran, S. Evolutionary history and stress regulation of the lectin superfamily in higher plants. BMC Evol. Biol. 2010, 10, 79. [CrossRef] [PubMed]

43. Kristan, K.Č.; GabriellaViero Serra, M.D.; Maček, P.; Anderluh, G. Molecular mechanism of pore formation by actinoporins. Toxicon 2009, 54, 1125-1134. [CrossRef] [PubMed]

44. DeLano, W.L. The PyMOL Molecular Graphics System; Delano Scientific LLC: Palo Alto, CA, USA, 2008.

45. Varrot, A.; Basheer, S.M.; Imberty, A. Fungal lectins: Structure, function and potential applications. Curr. Opin. Struct. Biol. 2013, 2013, 678-685. [CrossRef] [PubMed]

46. Ismaya, W.T.; Rozeboom, H.J.; Schurink, M.; Boeriu, C.G.; Wichers, H.; Dijkstra, B.W. Crystallization and preliminary X-ray crystallographic analysis of tyrosinase from the mushroom Agaricus bisporus. Acta Cryst. 2011, F67, 575-578.

47. Flurkey, A.; Cooksey, J.; Reddy, A.; Spoonmore, K.; Rescigno, A.; Inlow, J.; Flurkey, W.H. Enzyme, protein, carbohydrate, and phenolic contaminants in commercial tyrosinase preparations: Potential problems affecting tyrosinase activity and inhibition studies. J. Agric. Food Chem. 2008, 56, 4760-4768. [CrossRef]

48. Fujii, Y.; Gerdol, M.; Hasan, I.; Koide, Y.; Matsuzaki, R.; Ikeda, M.; Rajia, S.; Ogawa, Y.; Ozeki, Y. Phylogeny and properties of a novel lectin family with $\beta$-trefoil folding in mussels. Trends Glycosci. Glyc. 2018, 30, E195-E208. [CrossRef]

49. Nabila, N.; Meidianto, V.F.; Tjandrawinata, R.R.; Rachmawati, H.; Ismaya, W.T. Agaricus bisporus mannose binding protein is not an agglutinating protein. Biochem. Biophys. Res. Commun. 2019, 519, 773-776. [CrossRef]

50. Ismaya, W.T.; Yunita Damayanti, S.; Caroline Tjandrawinata, R.R.; Retnoningrum, D.S.; Rachmawati, H. In silico study to develop a lectin-like protein from mushroom Agaricus bisporus for pharmaceutical application. Sci. Pharm. 2016, 84, 203-217. [CrossRef]

51. Lai, X.; Soler-Lopez, M.; Ismaya, W.T.; Wichers, H.J.; Dijkstra, B.W. Crystal structure of recombinant MtaL at 1.35 Angstrom resolution. Acta Crystallogr. 2016, F72, 244-250.

52. Miyashita, S.-I.; Sagane, Y.; Suzuki, T.; Matsumoto, T.; Niwa, K.; Watanabe, T. "Non-Toxic" Proteins of the Botulinum Toxin Complex Exert In-vivo Toxicity. Sci. Rep. 2016, 6, 31043. [CrossRef]

53. Nosanchuk, J.D.; Casadevall, A. Impact of Melanin on Microbial Virulence and Clinical Resistance to Antimicrobial Compounds. Antimicrob. Agents Chemother. 2006, 50, 3519-3528. [CrossRef]

54. Yu, L.; Fernig, D.G.; Smith, J.A.; Milton, J.D.; Rhodes, J.M. Reversible inhibition of proliferation of epithelial cell lines by Agaricus bisporus (edible mushroom) lectin. Cancer Res. 1993, 153, 4627-4632.

55. Parslew, R.; Jones, K.; Rhodes, J.; Sharp, G.R. The antiproliferative effect of lectin from the edible mushroom (Agaricus bisporus) on human keratinocytes: Preliminary studies on its use in psoriasis. Br. J. Dermatol. 1999, 140, 56-60. [CrossRef]

56. Batterbury, M.; Tebbs, C.A.; Rhodes, J.M.; Grierson, I. Agaricus bisporus (edible mushroom lectin) inhibits ocular fibroblast proliferation and collagen lattice contraction. Exp. Eye Res. 2002, 74, 361-370. [CrossRef] 
57. Cheung, Y.-H.; Sheridan, C.M.; Lo, A.C.Y.; Lai, W.W. Lectin from Agaricus bisporus Inhibited S Phase Cell Population and Akt Phosphorylation in Human RPE Cells. Invest. Ophthalmol. Vis. Sci. 2012, 53, 7469-7475. [CrossRef]

58. Wang, Y.; Liu, Y.; Wang, H.; Li, C.; Qi, P.; Bao, J. Agaricus bisporus lectins mediates islet $\beta$-cell proliferation through regulation of cell cycle proteins. Experiment. Biol. Med. 2012, 237, 287-296. [CrossRef]

59. Wang, H.X.; Ng, T.B. Examination of lectins, polysaccharopeptide, polysaccharide, alkaloid, coumarin and trypsin inhibitors for inhibitory activity against human immunodeficiency virus reverse transcriptase and glycohydrolases. Planta Med. 2001, 67, 669-672. [CrossRef]

60. Greene, W.; Fleisher, T.; Waldmann, T. Suppression of human T and B lymphocyte activation by Agaricus bisporus lectin. I. Suggestive evidence for a surface "suppressor" receptor in human lymphocytes. J. Immunol. 1981, 126, 580-586.

61. Ditamo, Y.; Rupil, L.L.; Sendra, V.G.; Nores, G.A.; Roth, G.A.; Irazoqui, F.J. In vivo immunomodulatory effect of the lectin from edible mushroom Agaricus bisporus. Food Funct. 2016, 7, 262-269. [CrossRef]

62. Diana, D.; Ismaya, W.T.; Meidianto, V.F.; Tandrasasmita, O.M.; Tjandrawinata, R.R.; Rachmawati, H. Bioconjugation of captopril-light subunit of Agaricus bisporus mushroom tyrosinase: Characterization and potential use as a drug carrier for oral delivery. Biol. Pharm. Bull. 2018, 41, 1837-1842. [CrossRef]

63. Haltner, E.; Easson, J.H.; Lehr, C.-M. Lectins and bacterial invasion factors for controlling endo- and transcytosis of bioadhesive drug carrier systems. Eur. J. Pharm. Biopharm. 1997, 44, 3-13. [CrossRef]

64. Ismaya, W.T.; Efthyani, A.; Retnoningrum, D.S.; Lai, X.; Dijkstra, B.W.; Tjandrawinata, R.R.; Rachmawati, H. Study of response of Swiss Webster mice to light subunit of mushroom tyrosinase. Biotech. Histochem. 2017, 92, 411-416. [CrossRef]

65. Ismaya, W.T.; Efthyani, A.; Tjandrawinata, R.R.; Rachmawati, H. Biological responses in Balb/c mice after long-term parenteral administration of the light subunit of mushroom tyrosinase. J. Biochem. Mol. Toxicol. 2017, 31, e21958. [CrossRef]

66. Mazalovska, M.; Kouokam, J.C. Lectins as promising therapeutics for the prevention and treatment of HIV and other potential coinfections. BioMed. Res. Int. 2018, 2018, 3750646-3750657. [CrossRef]

67. Yau, T.; Dan, X.; Ng, C.C.W.; Ng, T.B. Lectins with potential for anti-cancer therapy. Molecules. 2015, 20, 3791-3810. [CrossRef]

68. Hoe, K.K.; Verma, C.S.; Lane, D.P. Drugging the p53 pathway: Understanding the route to clinical efficacy. Nat. Rev. Drug Discov. 2014, 13, 217-236.

69. Ye, Z.; Zhang, Q.; Wang, S.; Bharate, P.; Varela-Aramburu, S.; Lu, M.; Seeberger, P.H.; Yin, J. Tumour-targeted drug delivery with mannose-functionalized nanoparticles self-assembled from amphiphilic b-cyclodextrins. Chem. Eur. J. 2016, 22, 15216-15221. [CrossRef]

70. Jamieson, D.; Sunter, N.; Muro, S.; Pouche, L.; Cresti, N.; Lee, J.; ludden, J.; Griffin, M.J.; Allan, J.M.; Verrill, M.W.; et al. Pharmacogenetic association of MBL2 and CD95 polymorphisms with grade 3 infection following adjuvant therapy for breast cancer with doxorubicin and cyclophosphamide. Eur. J. Cancer. 2017, 71, 15-24. [CrossRef]

71. Kang, H.; Driscoll, H.; Mammoto, A.; Watters, A.L.; Melakeberhan, B.; Diaz, A.; Super, M.; Ingber, D.E. An engineered human Fc-mannose-binding-lectin captures circulating tumor cells. Adv. Biosys. 2017, 1, 1700094-1700100. [CrossRef]

(C) 2020 by the authors. Licensee MDPI, Basel, Switzerland. This article is an open access article distributed under the terms and conditions of the Creative Commons Attribution (CC BY) license (http://creativecommons.org/licenses/by/4.0/). 